\title{
The effect of hydrostatic pressure on the operation of quantum cascade lasers
}

\author{
Alfred R. Adams ${ }^{\mathrm{a}}$, Igor P. Marko ${ }^{\mathrm{a}}$, and Stephen J. Sweeney ${ }^{\mathrm{a} *}$, \\ Roland Teissier ${ }^{\mathrm{b}}$, Alexei N. Baranov ${ }^{\mathrm{b}}$, and Stanko Tomićc \\ a Advanced Technology Institute and Department of Physics, \\ University of Surrey, Guildford, Surrey, GU2 7XH, UK; \\ ${ }^{\mathrm{b}}$ Institut d'Électronique du Sud UMR5214 CNRS/Université Montpellier 2, \\ 34095 Montpellier, France; \\ ${ }^{c}$ Computational Science and Engineering Department, STFC Daresbury Laboratory, \\ Cheshire WA4 4AD, UK
}

\begin{abstract}
Quantum Cascade Lasers (QCLs) have been very successful at long wavelengths, $>4 \mu \mathrm{m}$, and there is now considerable effort to develop QCLs for short wavelength $(2-3 \mu \mathrm{m})$ applications. To optimise both interband and QC lasers it is important to understand the role of radiative and non-radiative processes and their variation with wavelength and temperature. We use high hydrostatic pressure to manipulate the band structure of lasers to identify the dominant efficiency limiting processes. We describe how hydrostatic pressure may also be used to vary the separation between the $\Gamma, \mathrm{X}$ and L bands, allowing one to investigate the role of inter-valley carrier scattering on the properties of QCLs. We

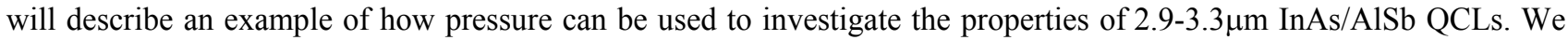
find that while the threshold current of the $3.3 \mu \mathrm{m}$ devices shows little pressure variation even at room temperature, for the $2.9 \mu \mathrm{m}$ devices the threshold current increases by $\sim 20 \%$ over $4 \mathrm{kbar}$ at $190 \mathrm{~K}$ consistent with carrier scattering into the L-minima. Based on our high pressure studies, we conclude that the maximum operating temperature of InAs/AlSb QCLs decreases with decreasing wavelength due to increased carrier scattering into the L-minima of InAs.
\end{abstract}

Keywords: quantum cascade laser, high hydrostatic pressure, carrier leakage, InAs/AlSb, non-radiative processes.

\section{INTRODUCTION}

Producing lasers for the mid-infrared spectral region 3-4 $\mu \mathrm{m}$ is of key importance for gas sensing and free space optical communications. There are several strategies being investigated to cover this spectral range using inter-band quantum well or quantum cascade lasers. However the performance of interband lasers tends to deteriorate with increasing emission wavelength due to increasing non-radiative Auger recombination and optical losses. Quantum Cascade Lasers (QCLs) have been very successful above $4 \mu \mathrm{m}[1,2]$ and considerable effort is now being put into reducing the wavelength of QCLs to target the shorter wavelength applications [3, 4]. Potential advantages of QCLs are high temperature stability of the threshold current, $\mathrm{I}_{\mathrm{th}}$, (characterised by high $\mathrm{T}_{0}$ values: $\mathrm{T}_{0}=\mathrm{I}_{\mathrm{th}}\left(\mathrm{dI}_{\mathrm{th}} / \mathrm{dT}\right.$ ) ${ }^{-1}$ ) and high output power. Here we discuss short wavelength InAs/AlSb quantum cascade lasers emitting around $3 \mu \mathrm{m}$ [4].

Unlike interband lasers in the mid-infrared, QCLs are not limited by non-radiative Auger recombination, however, modelling suggests that the operation of short wavelength QCLs may be affected by electron leakage from the upper levels of the laser transition into energy states associated with indirect conduction band valleys. Compared with other materials used at present in QCLs, the InAs/AlSb system exhibits not only the highest conduction band offset $(2.1 \mathrm{eV})$, but also the largest separation of direct $(\Gamma)$ and indirect $(\mathrm{L})$ minima in the quantum well conduction band $(0.72-0.76 \mathrm{eV})$ [3]. To investigate the presence and importance of the L-leakage loss mechanism, we analysed the pressure dependencies of the lasing threshold current, $\mathrm{I}_{\mathrm{th}}$, in InAs/AlSb QCLs as a function of temperature. We use high hydrostatic pressure to manipulate the band structure of lasers to identify the dominant efficiency limiting processes. This was used to great effect in near- and mid-infrared interband lasers where varying the direct band gap of the devices with pressure was key

\footnotetext{
* Corresponding author: e-mail s.sweeney@surrey.ac.uk, Phone: +44 (0)1483689 406, Fax: +44 (0)1483 689404
}

Quantum Sensing and Nanophotonic Devices VI, edited by Manijeh Razeghi, Rengarajan Sudharsanan, Gail J. Brown, Proc. of SPIE Vol. 7222, 722203 - @ 2009 SPIE · CCC code: 0277-786X/09/\$18 · doi: 10.1117/12.814322 
in identifying the importance of Auger recombination and intervalence band absorption on device properties (e.g. see [5]). Here we describe how hydrostatic pressure may also be used to vary the separation between the $\Gamma, \mathrm{X}$ and $\mathrm{L}$ bands, allowing one to investigate the role of inter-valley carrier scattering on the properties of QCLs at a constant temperature.

\section{EXPERIMENTAL DETAILS}

QCLs emitting near $2.9 \mu \mathrm{m}$ and $3.3 \mu \mathrm{m}$ were grown by MBE and processed into ridge lasers (ridge widths of 12 and $17 \mu \mathrm{m})$ using wet chemical etching and standard photolithography. Lasers were cleaved in cavities with lengths of 1.1$2.05 \mathrm{~mm}$. Details of the growth and structure of the lasers can be found in $[3,4]$.

Devices were investigated in the temperature range of $80-295 \mathrm{~K}$ using a static gas exchange cryostat. Lasers were operated in pulsed mode with a repetition rate of $1 \mathrm{kHz}$ and pulse width of $100 \mathrm{~ns}$. The emission intensity was measured using a cooled InSb detector and lock-in amplifier. For the high pressure studies the lasers were mounted in the pressure cell, which was then placed in a liquid nitrogen cooled cryostat. The cell was connected to a helium gas compressor, which can generate pressures up to 10kbar. Further details of the experimental arrangement can be found elsewhere [6].

\section{RESULTS AND DISCUSSION}

Figure 1 shows the temperature dependence of the threshold current density for both of the QCLs. The laser emitting at $2.9 \mu \mathrm{m}$ exhibited a higher threshold current and lower maximum operation temperature, $\mathrm{T}_{\max }$, compared with the longer wavelength device. The threshold current density of a QCL at its maximum operation temperature corresponds to it entering into the negative differential resistance (NDR) region of the current-voltage characteristics which indicates misalignment of energy levels in the active region at high electric field resulting in switching the laser action off. It should be noted that by using longer cavities $(\sim 4 \mathrm{~mm})$ it was possible to reach a high $\mathrm{T}_{\max }$ of $280 \mathrm{~K}$ and $400 \mathrm{~K}$ for the $2.9 \mu \mathrm{m}$ and $3.3 \mu \mathrm{m}$ devices, respectively (see later and $[3,4]$ ). The difference in $\mathrm{T}_{\max }$ may indicate that a temperature sensitive loss process in these lasers is more important at shorter wavelengths, which is in agreement with the assumption of indirect carrier leakage into the L minimum of the conduction band, since the shorter wavelength devices have a smaller $\Gamma$-L separation.

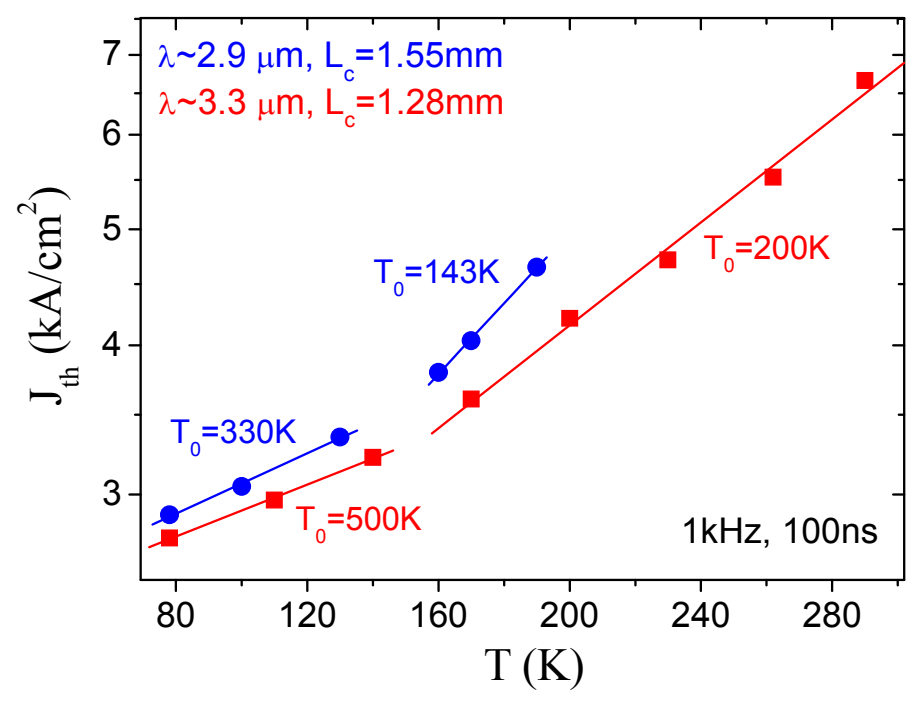

Fig. 1. Temperature dependence of threshold current density of the two InAs/AlSb based QCLs with emission wavelengths near $2.9 \mu \mathrm{m}$ and $3.3 \mu \mathrm{m}$. 
High hydrostatic pressure results provide further evidence that carrier scattering between the $\Gamma$ and $\mathrm{L}$ valleys plays a significant role in operation of short-wavelength QCLs. Figure 3 shows the pressure dependence of $\mathrm{I}_{\text {th }}$ at different temperatures for two such QC lasers with different lasing wavelengths. We assume that the total threshold current is formed by two components: current due to inter-subband longitudinal optical phonon scattering, $\mathrm{I}_{\mathrm{ph}}$, and current loss due to the leakage into the L-valleys, $I_{\text {leak }}^{L}$. Compared to phonon-scattering, the radiative lifetime is relatively long, meaning that the current component related to radiative transitions is very small in QCLs at threshold and hence is neglected in this analysis. The dashed lines $\left(\mathrm{I}_{\mathrm{ph}}\right)$ in Figure 2 describe the pressure dependence of inter-subband carrier relaxation via longitudinal polar optical phonon scattering calculated using the Frölich Hamiltonian approximation [7].

The theoretical pressure variation of the leakage current is also plotted in the figure for different temperatures and was derived using the temperature dependence of the leakage current at threshold which may be calculated from Eq. 1 [8]:

$$
I_{\text {leak }}^{L}(T)=I_{\text {0leak }} \exp \left(-\frac{E_{a}}{k T}\right)
$$

where $I_{\text {oleak }}$ is a constant, $k$ is the Boltzmann constant, and $E_{a}$ is the activation energy of the leakage process, which is equal to the energy separation between the quasi-Fermi level (assumed to be at the upper lasing level) and L minimum of the InAs quantum well. Using (1) we can write the following expression:

$$
I_{\text {leak }}^{L}(p) \propto \exp \left(-\left(\frac{d E_{L}}{d p}-\frac{d E_{\Gamma}}{d p}\right) \frac{p}{k T}\right)
$$

where $p$ is pressure value, $d E_{L} / d p$ and $d E_{\Gamma} / d p$ are pressure coefficients of the direct $\Gamma$ and indirect $\mathrm{L}$ band gaps in the InAs quantum wells. We assume here that $d E_{a}=d E_{L}-d E_{\Gamma}$. Taking into account the pressure dependence of the $\Gamma$ and $\mathrm{L}$ minima (with increasing pressure the energy separation between the $\Gamma$ and $\mathrm{L}$ minima decreases at a rate of $\sim 7 \mathrm{meV} / \mathrm{kbar}$ ) [9] and using (2) we can estimate the pressure dependence of $\Gamma$-L carrier leakage.

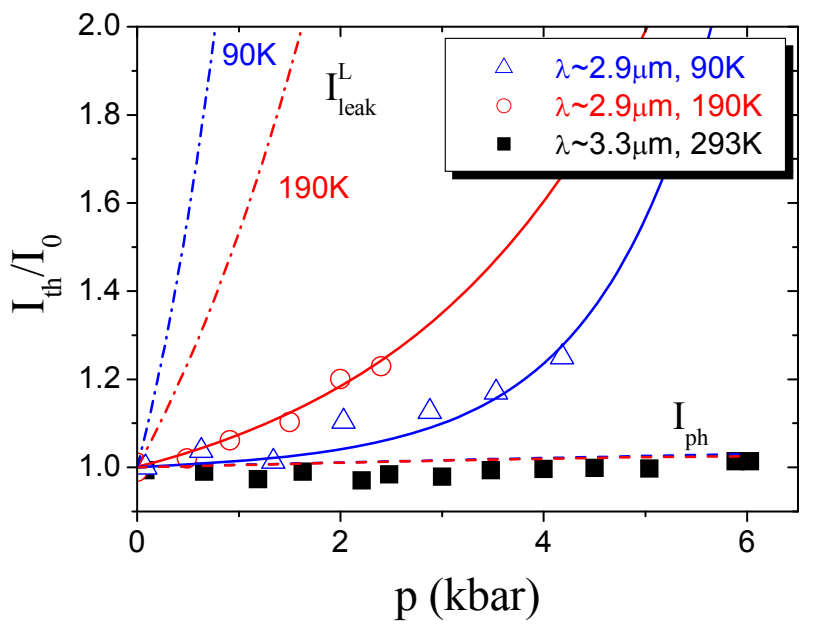

Fig. 2. Symbols show experimental dependencies of the normalised threshold current to its value at atmospheric pressure, $\mathrm{I}_{0}$, measured for the two QCLs at different temperatures $(2.9 \mu \mathrm{m}$ device with cavity length of $2.05 \mathrm{~mm}$ and $3.3 \mu \mathrm{m}$ device with cavity length of $1.28 \mathrm{~mm}$ ). The dashed lines present theoretical calculations of the pressure dependence of intersubband carrier relaxation via longitudinal polar optical phonon scattering in the shorter wavelength device. Dashdotted lines show the current due to the carrier leakage into the L subband calculated from equation (2). The solid lines are a fit to the data points using (3) and $\mathrm{x}=0.006$ at $90 \mathrm{~K}$ and $\mathrm{x}=0.13$ at $190 \mathrm{~K}$.. 
One can see from Fig.2 that for the $3.3 \mu \mathrm{m}$ QC laser, $\mathrm{I}_{\mathrm{th}}$ is almost pressure independent, which shows that carrier leakage into the L-valleys is not detectable in this device even at RT. The threshold current in this case is due to phonon scattering intersubband relaxation with very weak pressure dependence due to a small pressure dependence of the phonon energy. The pressure dependence of intersubband carrier relaxation via longitudinal polar optical phonon scattering calculated for the $2.9 \mu \mathrm{m}$ device is shown in Fig. 2. However, in contrast, the measured threshold current of this device increases considerably with pressure indicating significant leakage into the L-valley. This effect becomes more pronounced with increasing temperature (see results for $90 \mathrm{~K}$ and $190 \mathrm{~K}$ in Figure 2) in accordance with (1). The effect of pressure is approximately the same at $3.8 \mathrm{kbar}, 90 \mathrm{~K}$ and at only $2.1 \mathrm{kbar}$ at $190 \mathrm{~K}$. It was not possible to achieve lasing above approximately 2.4 kbar and $4.2 \mathrm{kbar}$ at $190 \mathrm{~K}$ and $90 \mathrm{~K}$, respectively.

To quantify value of the carrier leakage in $2.9 \mu \mathrm{m}$ QCLs we fit experimental points in Figure 3 taking into account that:

$$
\frac{I_{t h}}{I_{0}}=(1-x) \cdot \frac{I_{p h}}{I_{p h 0}}+x \cdot \frac{I_{\text {leak }}^{L}}{I_{\text {leak } 0}^{L}},
$$

where $\mathrm{I}_{0}, \mathrm{I}_{\text {pho }}$, and $\mathrm{I}_{\text {eak } 0} \mathrm{~L}$ are the threshold current, phonon scattering current and leakage current at atmospheric pressure, respectively. $x$ is a fraction of $\mathrm{I}_{0}$ due to leakage into the L-minima at atmospheric pressure. The solid lines in Figure 2 are the fitting curves for the data at $90 \mathrm{~K}$ and $190 \mathrm{~K}$. A good fit is obtained if we assume that the loss of current due to Lleakage increases from $0.6 \%$ of $\mathrm{I}_{\mathrm{th}}$ at $90 \mathrm{~K}$ up to $13 \%$ of $\mathrm{I}_{\mathrm{th}}$ at $190 \mathrm{~K}$. By applying pressure we increase the leakage current up to $29 \%$ of $\mathrm{I}_{\mathrm{th}}$ at $2.4 \mathrm{kbar}$ at $190 \mathrm{~K}$ and up to $20 \%$ of $\mathrm{I}_{\mathrm{th}}$ at $4.2 \mathrm{kbar}$ at $90 \mathrm{~K}$. Using absolute values of the leakage current determined for the $2.9 \mu \mathrm{m}$ device from the fit in Figure 3 at 90 and $190 \mathrm{~K}$, together with equation (1) we estimate that $E_{a} \approx 48 \mathrm{meV}$, which is in reasonable agreement with the expected value [3].

Figure 3 shows light-current characteristics of the $2.9 \mu \mathrm{m}$ laser measured as a function of pressure at $90 \mathrm{~K}$. One can see that the increase in the threshold current with pressure is accompanied by a decrease in the current corresponding to the maximum of the light intensity (maximum available current, $\mathrm{I}_{\max }$ ). The characteristics of this laser at $190 \mathrm{~K}$ were similar but the effect of the pressure on $\mathrm{I}_{\mathrm{th}}$ was stronger than on $\mathrm{I}_{\max }$. For a given design the maximum available current in a QCL is determined by the total rate of supply of electrons in the structure, i.e. by the injector doping density. After alignment of the structure above the turn-on voltage, the current grows quickly with applied voltage because of the fast supply of electrons from the doped injector. When the supply of electrons from the injector becomes limited at $I_{\max }$, the small increase in current leads to a large increase in the electric field, which results in misalignment of the structure and ceasing of the laser action. The threshold current of the laser grows with pressure because of the reduction of population of the upper level of the lasing transition due to the leakage into the L-valleys. As the electron lifetime in the L-valley associated states is expected to be longer than in the $\Gamma$-levels due to the fact that the probability of indirect phonon assisted transitions is smaller than the probability of direct transitions via $\Gamma$-states, the maximum available current decreases for the same total number of electrons in the structure. 


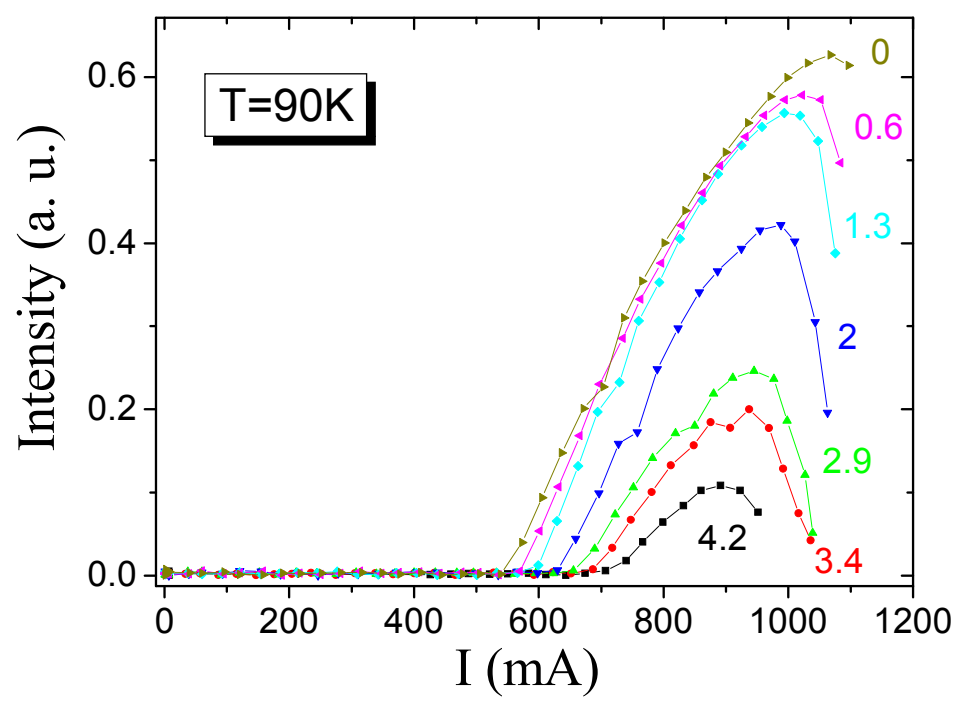

Fig. 3. Light-current characteristics of the $2.9 \mu \mathrm{m}$ laser measured at different pressures at $90 \mathrm{~K}$. The numbers near each curve shows the pressure value in kbar. NB: laser light was measured without collecting optics. Therefore the collection efficiency may vary with pressure. This does not affect our interpretation of the results.

Figure 4 shows maximum operation temperature of short wavelength InAs/AlSb QCLs as a function of emission wavelength [3]. These data were obtained on longer lasers, which is responsible for their higher operation temperatures compared with results of this work. We showed that operation of the $3.3 \mu \mathrm{m}$ QCLs is not affected by the electron leakage into the L-valley. It is possible to estimate now the influence of this loss on high temperature performances of the $2.9 \mu \mathrm{m}$ lasers. We used equation (1) and the $E_{a}$ value as determined above to quantify the loss due to $\Gamma$-L carrier leakage at the maximum temperature of $280 \mathrm{~K}$ as presented in [3]. Assuming that at $190 \mathrm{~K}$ the leakage current accounts up to $13 \%$ of $\mathrm{I}_{\text {th }}$ we found that at the maximum temperature the leakage current is about $20 \%$ of $\mathrm{I}_{\mathrm{th}}$. This relatively small contribution of the leakage to the total current suggests that the temperature performance of such QCLs can be improved through optimisation of the design to decrease the relative influence of leakage. For the QCLs emitting at $2.75 \mu \mathrm{m}$, the L-valley impact is certainly stronger in these devices and should be considered as an important factor limiting their performance. 


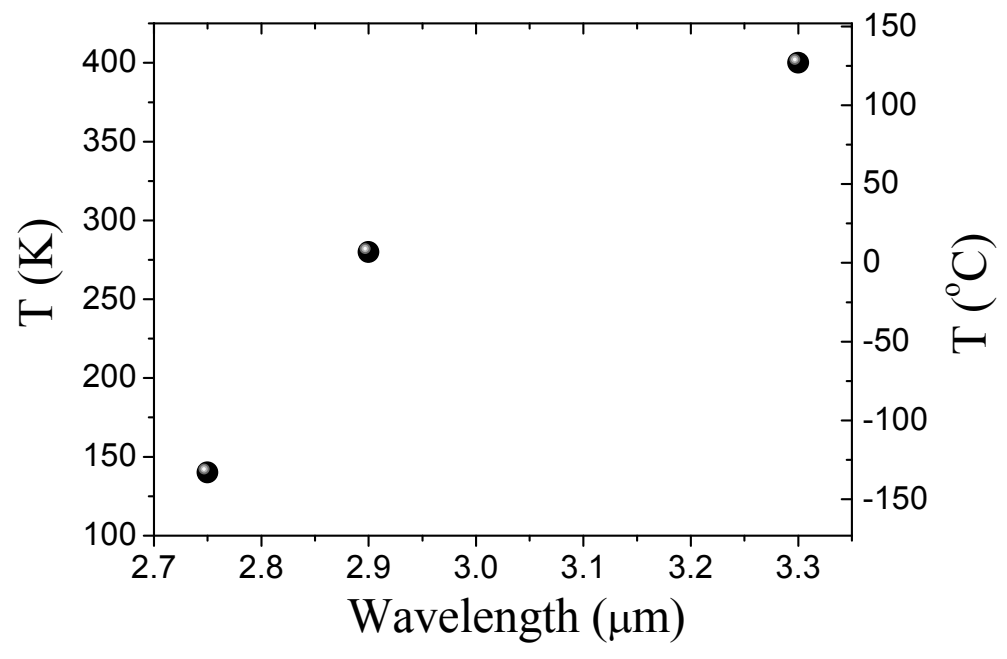

Fig. 4. Maximum operating temperature as a function of wavelength for InAs/AlSb QCLs [3].

\section{CONCLUSION}

To summarise, the impact of $\Gamma$-L scattering on the temperature performance of short wavelength InAs/AlSb QCLs was investigated using high hydrostatic pressure. The results show clear evidence of the presence of this loss mechanism in lasers with emission wavelength near $\sim 2.9 \mu \mathrm{m}$, whereas it is much less pronounced in the longer wavelength $\sim 3.3 \mu \mathrm{m}$ devices allowing their superior temperature performance. We suggest that increased leakage into the L-minima is the reason for the even lower operating temperature limit $(140 \mathrm{~K})$ of lasers emitting near $2.75 \mu \mathrm{m} \mathrm{[3].} \mathrm{In} \mathrm{order} \mathrm{to} \mathrm{further}$ reduce the operating wavelength of InAs/AlSb QCLs, carrier leakage into the indirect L valley should be minimised to achieve laser operation over an extended temperature range.

\section{REFERENCES}

[1] Y. Bai, S. R. Darvish, S. Slivken, W. Zhang, A. Evans, J. Nguyen, and M. Razeghi, Appl. Phys. Lett. 92, 101105 (2008).

[2] A. Lyakh, C. Pflügl, L. Diehl, Q. J. Wang, F. Capasso, X. J. Wang, J. Y. Fan, T. Tanbun-Ek, R. Maulini, A. Tsekoun, R. Go, and C. Kumar N. Patel, Appl. Phys. Lett. 92, 111110 (2008).

[3] J. Devenson, O. Cathabard, R. Teissier, A. N. Baranov, Appl. Phys. Lett. 91, 251102 (2007).

[4] J. Nguyen, Y. Bai, and M. Razeghi, Appl. Phys. Lett. 91, 141106 (2007).

[5] B. N. Murdin, A. R. Adams and S. J. Sweeney, in Mid-infrared Semiconductor Optoelectronics (Springer Series in Optical Sciences), Springer, ISBN 184628208X, May 2006.

[6] I.P. Marko, A.R. Adams, S.J. Sweeney, R. Teissier, A.N. Baranov, S. Tomic, Phys. Stat. Sol. (b) in press (2008).

[7] S. R. Jin, C. N. Ahmad, S. J. Sweeney, A. R. Adams, B. N. Murdin, H. Page, X. Marcadet, C. Sirtori, S. Tomić, Appl. Phys. Lett. 89, 221105 (2006).

[8] S. J. Sweeney, G. Knowles, T. E. Sale, and A. R. Adams, Phys. Stat. Sol. (b) 223, 567 (2001).

[9] E. H. Li, Physica E 5, 215 (2000); A. L. Edwards and H. G. Drickamer, Phys. Rev. 122, 1149 (1961). 